\title{
Ongoing Attention to Injurious Inpatient Falls and Pressure Ulcers
}

\section{Citation}

Lee, Grace M., and Stephen B. Soumerai. 2015. “Ongoing Attention to Injurious Inpatient Falls and Pressure Ulcers." JAMA Internal Medicine 175 (9) (September 1): 1581. doi:10.1001/ jamainternmed.2015.2552.

\section{Published Version}

doi:10.1001/jamainternmed.2015.2552

\section{Permanent link}

http://nrs.harvard.edu/urn-3:HUL.InstRepos:32696163

\section{Terms of Use}

This article was downloaded from Harvard University's DASH repository, and is made available under the terms and conditions applicable to Other Posted Material, as set forth at http:// nrs.harvard.edu/urn-3:HUL.InstRepos:dash.current.terms-of-use\#LAA

\section{Share Your Story}

The Harvard community has made this article openly available.

Please share how this access benefits you. Submit a story.

\section{Accessibility}


the dose-response association between sodium intake and outcomes. ${ }^{1,2}$ On the other hand, limited precision does dilute regression estimates, and this might have weakened the association with the outcomes of interest in our study. We also agree that statistical power was limited in the high sodium intake group. We categorized sodium intake using cutoff points driven by the current US recommendations to facilitate clinical interpretation. We would have liked to analyze a very high sodium intake subgroup, but the number of participants was too small to provide stable estimates. The small numbers in the very high sodium subgroup are potentially related to underestimation of sodium intake with FFQ. On the other hand, the dose-response association across the range of reported intakes is evident in the spline graph (Figure 1) provided in the main article. ${ }^{3}$

The point about potassium is important and supported by the recently reported findings from O'Donnell and colleagues. ${ }^{2}$ To address this comment, we evaluated self-reported potassium intake in our cohort. The median daily intake was 2750 $\mathrm{mg}$ (2130-3500 mg/d), which is comparable to other cohorts. ${ }^{2,4}$ However, we did not observe any significant association (either crude or adjusted) between self-reported potassium intake and risk of mortality, cardiovascular disease, or heart failure in the Health ABC Study (unpublished data). This may have to do with the limitations of self-reporting as discussed above, or with the fact that our cohort consists of considerably older participants compared with the aforementioned cohorts. ${ }^{2,4}$ Of note, sodium and potassium intakes were estimated using an FFQ in the study by Umesawa and colleagues. ${ }^{4}$

We agree that changing dietary habits over time could have led to regression dilution in our cohort. Without multiple dietary assessments, we are not able to rule this out. However, after explicit testing, we did not observe any time-dependent effects of baseline dietary sodium intake on study outcomes.

Finally, we agree that high sodium intake has important physiologic effects. However, the increase in monocytes observed by Yi and colleagues was evident only in the $12-\mathrm{g} / \mathrm{d}$ salt group, ${ }^{5}$ which corresponds to twice the amount of the currently recommended intake for the general population. Similarly, the high sodium intake group in the study by Zhou and colleagues ${ }^{6}$ was consuming $15 \mathrm{~g} / \mathrm{d}$ of salt. These data actually are in concordance with the aforementioned large-scale studies that observed association with harm only at dietary sodium intake levels exceeding $4000 \mathrm{mg} / \mathrm{d},{ }^{1,2}$ highlighting the need for prospective clinical trials to establish optimal sodium intake levels.

\section{Andreas P. Kalogeropoulos, MD, MPH, PhD Vasiliki V. Georgiopoulou, MD, MPH Stephen B. Kritchevsky, PhD}

Author Affiliations: Emory Clinical Cardiovascular Research Institute, Emory University, Atlanta, Georgia (Kalogeropoulos, Georgiopoulou); Section on Gerontology and Geriatric Medicine, Wake Forest School of Medicine, WinstonSalem, North Carolina (Kritchevsky)

Corresponding Author: Andreas P. Kalogeropoulos, MD, MPH, PhD, Emory Clinical Cardiovascular Research Institute, Emory University, 1462 Clifton Rd NE, Ste 535B, Atlanta, GA 30322 (akaloge@emory.edu)

Conflict of Interest Disclosures: None reported.
1. O'Donnell MJ, Yusuf S, Mente A, et al. Urinary sodium and potassium excretion and risk of cardiovascular events. JAMA. 2011;306(20):2229-2238.

2. O'Donnell M, Mente A, Rangarajan S, et al; PURE Investigators. Urinary sodium and potassium excretion, mortality, and cardiovascular events. N Engl J Med. 2014;371(7):612-623

3. Kalogeropoulos AP, Georgiopoulou VV, Murphy RA, et al. Dietary sodium content, mortality, and risk for cardiovascular events in older adults: the Health, Aging, and Body Composition (Health ABC) Study. JAMA Intern Med. 2015;175 (3):410-419.

4. Umesawa M, Iso $H$, Date $C$, et al; JACC Study Group. Relations between dietary sodium and potassium intakes and mortality from cardiovascular disease: the Japan Collaborative Cohort Study for Evaluation of Cancer Risks. Am J Clin Nutr. 2008;88(1):195-202.

5. Yi B, Titze J, Rykova M, et al. Effects of dietary salt levels on monocytic cells and immune responses in healthy human subjects: a longitudinal study. Transl Res. 2015;166(1):103-110.

6. Zhou X, Zhang L, Ji WJ, et al. Variation in dietary salt intake induces coordinated dynamics of monocyte subsets and monocyte-platelet aggregates in humans: implications in end organ inflammation. PLoS One. 2013;8(4):e60332. doi:10.1371/journal.pone.0060332.

\section{Ongoing Attention to Injurious Inpatient Falls and Pressure Ulcers}

To the Editor The effectiveness of Centers for Medicare \& Medicaid Services (CMS) performance-based payment strategies will shape efforts to improve the quality and value of care. Waters et $\mathrm{al}^{1}$ examined the effect of the Hospital-Acquired Conditions (HACs) Initiative, which denies incremental payment for 8 complications on 4 outcomes: central line-associated bloodstream infections (CLABSIs), catheter-associated urinary tract infections (CAUTIs), hospital-acquired pressure ulcers (HAPUs), and injurious inpatient falls. They found an association with reductions in CLABSIs and CAUTIs but not injurious falls or stage III/IV HAPUs. Waters et al concluded that there is less evidence that changing hospital processes affects these other 2 outcomes, but we believe this conclusion may be missing key factors.

Nursing leaders have advanced improvements in falls and HAPUs for nearly 2 decades. Central line-associated bloodstream infections and CAUTIs have more recently received attention and been added to National Healthcare Safety Network indicators. Waters et $\mathrm{al}^{1}$ report improvement in HAPU and fall rates prior to implementation of the HACs Initiative. This is consistent with prior reports using data from the Collaborative Alliance for Nursing Outcomes (CALNOC), the nation's first registry of nursing-sensitive outcomes data. In 2010, CALNOC reported improvements in HAPU rates from 2001 to $2008,{ }^{2}$ and in 2013, they reported continued improvements in HAPU rates from 2003 to $2010 .{ }^{3}$ Given that nursing leaders were steadily advancing improvements in these adverse outcomes, it is not surprising that the HACs Initiative had no additional effect.

The authors did not examine the prevalence of stage II HAPUs, even though the National Quality Forum endorses measures for stage II HAPUs and higher. ${ }^{4}$ Stage III/IV HAPUs are rare in most hospitals, with Waters et $\mathrm{al}^{1}$ reporting a 2010 rate of about $0.4 \%$, and CALNOC data reporting a similar rate that year, having dropped from $2.0 \%$ in $2003 .{ }^{3}$ The more common stage II HAPUs are clinically important, because appropriate interventions will prevent progression to stage III/IV. The 
analysis of only rare stage III/IV HAPUs may not detect important changes in prevention, identification, and treatment of HAPUs. Similarly, focusing only on injurious falls (also rare events) may fail to identify hospital mitigation of all falls. Substantial research finds that many interventions can improve rates of both falls and HAPUs. ${ }^{5}$

As stage III/IV HAPUs and injurious falls are eliminated, health care leaders should continue to focus on rates of all HAPUs and falls. There is ample evidence that continued improvement is feasible.

\section{Joanne Spetz, PhD \\ Diane S. Brown, PhD, RN \\ Carolyn S. Aydin, PhD}

Author Affiliations: Philip R. Lee Institute for Health Policy Studies, University of California, San Francisco (Spetz); Kaiser Permanente Northern California, Oakland (Brown); Collaborative Alliance for Nursing Outcomes (CALNOC), San Ramon, California (Brown, Aydin); Burns \& Allen Research Institute, CedarsSinai Medical Center, Los Angeles, California (Aydin).

Corresponding Author: Joanne Spetz, PhD, Philip R. Lee Institute for Health Policy Studies, University of California, San Francisco, 3333 California St, Ste 265, San Francisco, CA 94118 (joanne.spetz@ucsf.edu).

Conflict of Interest Disclosures: None reported.

1. Waters TM, Daniels MJ, Bazzoli GJ, et al. Effect of Medicare's nonpayment for hospital-acquired conditions: lessons for future policy. JAMA Intern Med. 2015; 175(3):347-354.

2. Brown DS, Donaldson N, Burnes Bolton L, Aydin CE. Nursing-sensitive benchmarks for hospitals to gauge high-reliability performance. J Healthc Qual. 2010;32(6):9-17.

3. Stotts NA, Brown DS, Donaldson NE, Aydin C, Fridman M. Eliminating hospital-acquired pressure ulcers: within our reach. Adv Skin Wound Care. 2013; 26(1):13-18.

4. National Quality Forum. National voluntary consensus standards for developing a framework for measuring quality for prevention and management of pressure ulcers. 2011.http://www.qualityforum.org/Publications/2011/12 /National_Voluntary_Consensus_Standards_for_Developing_a_Framework_for _Measuring_Quality_for_Prevention_and_Management_of_Pressure_Ulcers.aspx. Accessed July 1, 2015.

5. Aydin C, Donaldson N, Stotts NA, Fridman M, Brown DS. Modeling hospital-acquired pressure ulcer prevalence on medical-surgical units: nurse workload, expertise, and clinical processes of care. Health Serv Res. 2015;50(2): 351-373.

To the Editor We read with interest the article by Waters et $\mathrm{al}^{1}$ because we believe it is vital to evaluate whether the actual effect of payment policies matches their intended effect. The authors contribute unique information about the results of the Centers for Medicare \& Medicaid Services Hospital-Acquired Conditions Present on Admission Indicator (HAC POA) on pressure ulcers and injurious falls, using data from the National $\mathrm{Da}-$ tabase of Nursing Quality Indicators. However, their findings contradict those of our previously published study evaluating the impact of the HAC POA program on rates of central lineassociated bloodstream infections (CLABSIs) and catheterassociated urinary tract infections (CAUTIs). ${ }^{2}$ We continue this important conversation for the benefit of patients, clinicians, hospital leadership, and policymakers, and we raise the following key concerns with the goal of increasing transparency and ensuring that conclusions about the effect of the HAC POA program are robust.
First, Waters et $\mathrm{al}^{1}$ did not include a comparison group of conditions unaffected by the policy, which would have strengthened the study design for this policy evaluation and made it less prone to bias. A lack of comparison conditions limits the ability to interpret their conclusions. Second, the brief prepolicy period for CLABSIs and CAUTIs raises concerns about whether their preintervention trends estimated what would have happened in the absence of the HAC POA program, which is critical when making comparisons to postintervention trends. Third, Waters et al did not perform a sensitivity analysis based on hospitals that reported consistently throughout the study period. Thus, it is possible that the prepolicy trends may be driven by only a subset of the hospitals. Fourth, differences in data sources may contribute to differences in findings. Comparisons of data reported to the Centers for Disease Control and Prevention and to the National Database of Nursing Quality Indicators indicate meaningful differences, despite their use of the same definitions. ${ }^{3}$ Fifth, Waters et al speculate that differences in hospital characteristics might contribute to discordant findings, but they did not explore whether effect modification exists by region or teaching status in their study population. Finally, Waters et al did not show actual data and instead only displayed expected plots based on the model. Readers cannot effectively evaluate the model fit or understand how to interpret these findings without some representation of the data.

By increasing transparency on these issues, we can continue to contribute to national conversations about how to best improve quality of care and patient outcomes and better understand where future questions remain.

\section{Grace M. Lee, MD, MPH}

Stephen B. Soumerai, ScD; for the Preventing Avoidable Complications by Adjusting Payment (PAICAP) Study Team

Author Affiliations: Harvard Medical School, Boston, Massachusetts (Lee, Soumerai); Harvard Pilgrim Health Care Institute, Boston, Massachusetts (Lee, Soumerai).

Corresponding Author: Grace M. Lee, MD, MPH, Harvard Medical School \& Harvard Pilgrim Health Care Institute, 133 Brookline Ave, Sixth Flr, Boston, MA 02215 (grace.lee@childrens.harvard.edu).

Conflict of Interest Disclosures: None reported.

Funding/Support: Dr Lee received funding from the Agency for Healthcare Research and Quality (grant RO1HSO18414).

Role of the Funder/Sponsor: The Agency for Healthcare Research and Quality had no role in the preparation, review, or approval of the manuscript; and decision to submit the manuscript for publication.

PAICAP Study Team: Ken Kleinman, ScD; Michael Calderwood, MD, MPH

Additional Contributions: We thank our participating PAICAP hospitals for their contributions and the Agency for Healthcare Research and Quality for grant funding.

1. Waters TM, Daniels MJ, Bazzoli GJ, et al. Effect of Medicare's nonpayment for Hospital-Acquired Conditions: lessons for future policy. JAMA Intern Med. 2015; 175(3):347-354.

2. Lee GM, Kleinman K, Soumerai SB, et al. Effect of nonpayment for preventable infections in U.S. hospitals. N Engl J Med. 2012;367(15):1428-1437.

3. Health Services Advisory Group and Mathematica Policy Research. Project evaluation activity in support of partnership for patients: task 2 evaluation progress 
report. 2014. http://innovation.cms.gov/Files/reports/PFPEvalProgRpt.pdf. Accessed January 28, 2015

In Reply We agree with Lee and Soumerai that transparency is needed when evaluating payment policies to ensure that actual outcomes from a policy change match intended outcomes. The best way to ensure transparency is to conduct multiple evaluations, using different hospitals and databases. Deriving different results from a prior study by Lee et $\mathrm{al}^{1}$ does not necessarily mean our findings are wrong. Given the narrow confidence intervals around our findings and that we controlled for multiple hospital- and unit-level covariates, we believe the methodological concerns raised did not influence our findings. Rather, the location and teaching status of the 1381 hospitals contributing data to our study were quite different from the 398 hospitals in their study. The increased heterogeneity in our data likely provided more opportunity to identify the effects of the policy change.

In response to Lee and Soumerai, inclusion of conditions unaffected by the Hospital-Acquired Conditions (HAC) Initiative policy can offer insights on potential confounding only if conditions with no potential spillover effects from the HAC Initiative can be identified, which is quite difficult to assure. While our pre-HAC Initiative period for central line-associated bloodstream infections (CLABSIs) and catheter-associated urinary tract infections(CAUTIs) was just 9 months, we had monthly observations of more than 500 hospitals ensuring sufficient data to establish a trend. Also noted in our article, ${ }^{2}$ missing outcome data were rare, especially for CLABSIs and CAUTIs, making a sensitivity analysis for consistently reporting hospitals unnecessary. Because of our research focus and space constraints, we did not explore effect modification by organizational or regional characteristics. Instead, we chose to control linearly for these factors as main effects in our regressions. Finally, we are not sure what Lee and Soumerai would gain from reviewing actual data from the analysis. Table 2 of our article $^{2}$ reports odds ratios and incidence rate ratios across all the estimated models.

Spetz and colleagues asked why we did not examine the more common stage II hospital-acquired pressure ulcers (HAPUs). Although we acknowledge stage II HAPUs as prevalent and clinically meaningful, they were not targeted by the HAC Initiative. Therefore, we excluded them from our analysis. Because of potential HAC Initiative spillover effects on stage II HAPUs, a follow-up analysis could prove informative but was outside our article's scope. Our article and other reports show a consistent decline in injurious inpatient falls and HAPUs over time. While Spetz and colleagues were not surprised that the HAC Initiative had no additional effect on these trends, we hope our results stimulate further investigation and discussion.

Multiple empirical assessments combined with vigorous debate are vitally important for identifying key issues for improving quality of care and refining existing policies so that they support better health outcomes. We thank the commentators for thoroughly assessing our report and contributing to the ongoing discussion.

Teresa M. Waters, PhD

Michael J. Daniels, ScD

Gloria J. Bazzoli, PhD;

for the Hospital Responses to Medicare's Nonpayment for Preventable Complications (HRMNPC) Team

Author Affiliations: Department of Preventive Medicine, University of Tennessee Health Science Center, Memphis (Waters); Department of Statistics \& Data Sciences, The University of Texas at Austin, Austin (Daniels); Department of Integrative Biology, The University of Texas at Austin, Austin (Daniels); Department of Health Administration, Virginia Commonwealth University, Richmond (Bazzoli).

Corresponding Author: Teresa M. Waters, PhD, Department of Preventive Medicine, University of Tennessee Health Science Center, 66 N Pauline, Ste 633, Memphis, TN 38163 (twaters@uthsc.edu).

Conflict of Interest Disclosures: None reported.

HRMNPC Team: Eli Perencevich, MD; Nancy Dunton, PhD; Vincent S. Staggs PhD; Catima Potter, MPH; Naleef Fareed, PhD; Minzhao Liu, MS, PhD; and Ronald I. Shorr, MD, MS.

1. Lee GM, Kleinman $K$, Soumerai SB, et al. Effect of nonpayment for preventable infections in U.S. hospitals. N Engl J Med. 2012;367(15):1428-1437. 2. Waters TM, Daniels MJ, Bazzoli GJ, et al. Effect of Medicare's nonpayment for Hospital-Acquired Conditions: lessons for future policy. JAMA Intern Med. 2015; 175(3):347-354

\section{Prescription Opioid Duration of Action and the Risk of Unintentional Overdose}

To the Editor It is with great interest that we read the article "Prescription Opioid Duration of Action and the Risk of Unintentional Overdose Among Patients Receiving Opioid Therapy" published in the April issue of JAMA Internal Medicine. ${ }^{1}$ Miller and colleagues used data from the Veterans Administration Healthcare system and found that patients initiating opioid therapy with long-acting opioids were more than twice as likely to overdose compared with those initiating therapy with shortacting opioids.

We believe the authors failed to recognize that their results may be driven by use of the fentanyl patch, which should never be prescribed to an opioid-naïve patient. Out of 18887 opioid-naïve patients initiated with long-acting opioids, 4802 were started on the fentanyl patch, representing $25 \%$ of those starting long-acting opioids. The package insert of the fentanyl patch ${ }^{2}$ states clearly that this drug is contraindicated in patients who are opioid-nontolerant. We wonder what proportion of the patients receiving long-acting opioids who experienced overdose events were initiated to treatment with the fentanyl patch. We also believe the statement made by Miller and colleagues that their findings provide the first evidence that the risk of unintentional overdose injury is related to the prescribed opioid's duration of action may be overstated. Long-acting opioids provide sustained analgesia by way of gradually releasing the opioid for absorption, prolonging its analgesia. Sustained-release morphine represented over $40 \%$ of those starting long-acting opioids. However, under certain conditions (eg, when coingested with alcohol or taken with a high-fat meal), the slow-release delivery mechanism can be disrupted, dumping the opioid 